\title{
Three-dimensional kinematics of the human metatarsophalangeal joint during level walking
}

\section{Sivangi Raychoudhury, Dan Hu and Lei Ren*}

School of Mechanical, Aerospace and Civil Engineering, University of Manchester, Manchester, UK

Edited by:

Jill L. McNitt-Gray, University of

Southern California, USA

\section{Reviewed by:}

Henrique De Amorim Almeida,

Polytechnic Institute of Leiria, Portugal

Eiji Tanaka, The University of

Tokushima Graduate School, Japan

\section{*Correspondence:}

Lei Ren, School of Mechanical,

Aerospace and Civil Engineering,

University of Manchester,

Manchester M13 9PL, UK

e-mail: lei.ren@manchester.ac.uk
The objective of this study is to investigate the three-dimensional (3D) kinematics of the functional rotation axis of the human metatarsophalangeal (MP) joint during level walking at different speeds. A 12 camera motion analysis system was used to capture the 3D motion of the foot segments and a six force plate array was employed to record the simultaneous ground reaction forces and moments. The 3D orientation and position of the functional axis (FA) of the MP joint were determined based on the relative motion data between the tarsometatarsi (hindfoot) and phalanges (forefoot) segments. From the results of a series of statistical analyses, it was found that the FA remains anterior to the anatomical axis (AA), defined as a line connecting the first and fifth metatarsal heads, with an average distance about $16 \%$ of the foot length across all walking speeds, and is also superior to the AA with an average distance about $2 \%$ of the foot length during normal and fast walking, whereas the FA shows a higher obliquity than the AA with an anteriorly more medial and superior orientation. This suggests that using the AA to represent the MP joint may result in overestimated MP joint moment and power and also underestimated muscle moment arms for MP extensor muscles. It was also found that walking speed has statistically significant effect on the position of the FA though the FA orientation remains unchanged with varying speed. The FA moves forwards and upwards toward a more anterior and more superior position with increased speed. This axis shift may help to increase the effective mechanical advantage of MP extensor muscles, maximize the locomotor efficiency, and also reduce the risk of injury. Those results may further our understanding of the contribution of the intrinsic foot structure to the propulsive function of the foot during locomotion at different speeds.

Keywords: metatarsophalangeal joint, three-dimensional kinematics, position and orientation, walking, ground reaction force

\section{INTRODUCTION}

The human foot is an enormously complex structure consisting of numerous bones, muscles, ligaments, and synovial joints. As the only body component in contact with the ground, it plays multiple crucial roles in attenuating ground impacts, maintaining locomotor stability, and generating propulsive powers during locomotion (Ker et al., 1987; Carrier et al., 1994; Ren et al., 2008a). Over the past decades, many experimental and computer simulation studies have been conducted to investigate the locomotor function of the human foot complex (Apkarian et al., 1989; Scott and Winter, 1993; Leardini et al., 1999; Gefen et al., 2000; Carson et al., 2001; MacWilliams et al., 2003; Nester et al., 2007; Ren et al., 2010; Qian et al., 2013). However, most of those studies have mainly concentrated on the biomechanics of the whole foot segment, whereas the specific functioning of the distal part of the foot has been much less frequently studied.

The metatarsophalangeal (MP) joint near the distal end of the foot may have multiple functions during locomotion (BojsenMøller and Lamoreux, 1979; Mann and Hagy, 1979; Stefanyshyn and Nigg, 1997). During walking, the MP joint undergoes progressive dorsiflexion in the stance phase, which tightens up the plantar aponeurosis to wrap around the metatarsal heads. This may help to elevate and stabilize the longitudinal arch of the foot by using the windlass mechanism of the plantar aponeurosis without muscle function (Mann and Hagy, 1979). The dorsiflexion of the MP joint in the late stance phase can noticeably reduce the moment arm of the ground reaction force compared to a single rigid foot lever. This may increase the effective mechanical advantage (EMA) of ankle dosriflexor muscles and hence reduce the muscular effort during push-off (Bojsen-Møller and Lamoreux, 1979; Biewener, 1989). In addition, it was found that the dorsiflexion of the MP joint tightens the connective tissue framework around the ball of the foot, and thereby constraints the relative motions of the skin to enable shear forces to be transmitted to the skeleton (Bojsen-Møller and Lamoreux, 1979).

The MP joint may play significant roles during rapid change of body movement because the toes help in balancing the body while the body is changing its motion rapidly (Mann and Hagy, 1979). It was found that the MP joint is a significant absorber of energy in sprinting, with increased energy absorption with increased speed (Stefanyshyn and Nigg, 1997). Furthermore, it was suggested that ankle and MP joints may provide a mechanism of varying the gear ratio of the ankle dorsiflexor muscles during running (Carrier et al., 1994). This may enhance locomotor performance during 
constant speed running by maintaining the muscles near the high-efficiency portion of the force-velocity curve.

To simplify the analyses, many of the previous studies have assumed that the MP joint rotates about an axis perpendicular to the sagittal plane, originating from the fifth metatarsal head (Stefanyshyn and Nigg, 1997, 1998). This two-dimensional assumption simplifies the motion of the MP joint and does not reflect the oblique nature of the MP joint. A recent study investigated the effect of the MP joint axis by comparing the calculated joint kinetic variables based on different MP joint axis definitions in sprinting (Smith et al., 2012). It was found that MP joint axis has a significant effect on calculated joint moment, power, and energy, and oblique joint axes result in less energy absorbed at the MP joint than a simplified perpendicular axis to the sagittal plane. It was suggested that an appropriate representation of the MP joint is necessary to better understand the MP joint during locomotion (Smith et al., 2012). However, most previous studies defined the MP joint axis mainly based on anatomical landmarks, e.g., the straight line connecting first and fifth metatarsal heads (Bojsen-Møller and Lamoreux, 1979; Smith et al., 2012). So far, little is known about the realistic orientation and position of the functional rotation axis of the MP joint during locomotion and how it changes with varying locomotor speed (Pohl et al., 2007).

The objective of this study is to investigate the threedimensional (3D) kinematics of the human MP joint during level walking. The latest three-dimensional motion analysis technique was used to determine the position and orientation of the functional rotation axis of the MP joint in the stance phase of walking for multiple subjects at different speeds. Statistical analysis was conducted to evaluate the difference between the functional MP joint axis and the axis typically defined by the anatomical landmarks in literature. Moreover, the effect of walking speed on MP joint position and orientation was also analyzed statistically. This would provide useful information to improve our understanding of the in vivo biomechanical functioning of the human MP joint as well as the propulsive function of the foot during different speeds of locomotion. Furthermore, this may also help in innovating the design of sports and therapeutic footwear, prosthetic lower limbs, and robotic legs, which could be inspired from the nature design of the musculoskeletal system of the human body (Ren et al., 2014).

\section{MATERIALS AND METHODS GAIT MEASUREMENT}

Six healthy male subjects with normal foot conformation (age: $26.67 \pm 2.69$ years; weight: $67.17 \pm 10.29 \mathrm{~kg}$; height: $175.0 \pm 4.43 \mathrm{~cm}$ ) from a population of postgraduate students, with no previous medical history of foot and lower limb injury, participated in the gait measurement in this study. The subjects provided informed consent in accordance with the policies of local institute ethical advisory committee. All the subjects were instructed to walk barefoot along an indoor walkway at their self-selected slow, normal, and fast walking speeds. A specially designed marker cluster system was mounted firmly on the right feet of the subjects to record the $3 \mathrm{D}$ segmental motions of the foot complex (see Figure 1).

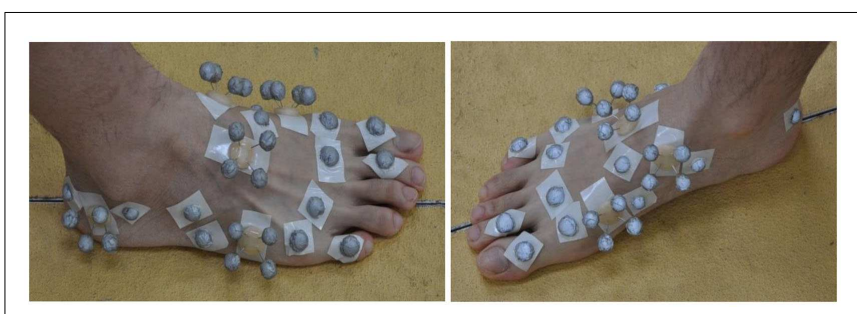

FIGURE 1 |The infrared marker cluster system used in this study to capture 3D foot motions. The foot was divided into two segments including tarsometatarsi (hindfoot) and phalanges (forefoot). A set of thermal plastic plates, each carrying four infrared markers, were used to capture the foot segmental motions. A number of hemispherical infrared markers were also attached on the anatomical landmarks.

A 12 infrared camera motion analysis system (Qualisys, Sweden) was used to capture 3D segmental motions at $150 \mathrm{~Hz}$. Six force plates (Kistler, Switzerland) were used to record the simultaneous ground reaction forces and moments at $1000 \mathrm{~Hz}$. A set of static calibration procedures were undertaken to locate the anatomical landmarks using a calibration wand and reflective markers according to the calibrated anatomical system technique (Cappozzo et al., 1995). The calibration markers were removed before the dynamic walking trials. For each walking speed, the measurement was repeated 15 times to ensure that representative walking data are recorded.

\section{MP JOINT DEFINITION AND PARAMETERS}

Five rigid body segments were defined to represent the lower limb: pelvis, right thigh, right shank, right tarsometatarsi (hindfoot), and right phalanges (forefoot). The 3D anatomical coordinate systems were defined for each individual segment based on the previous studies (Jenkyn and Nicol, 2007; Ren et al., 2008a, 2010). In this study, we have assumed that the five phalanges form a single rigid forefoot segment, whereas the MP joint is considered as a single hinge type joint. The anatomical axis (AA) of the MP joint was defined as the oblique line connecting the first metatarsal head and the fifth metatarsal head (see the blue line in Figure 2A), which is similar to those defined in previous studies (Boonpratatong and Ren, 2010; Graf et al., 2012; Smith et al., 2012). This axis divides the foot into two different segments: hindfoot and forefoot. On the other hand, the functional axis (FA) of the MP joint was defined as the rotational axis between the hindfoot segment and forefoot segment during stance phase of walking (see the red line in Figure 2A). A closed-form algorithm is employed to determine the 3D position and orientation of the FA of the MP joint (Gamage and Lasenby, 2002), which does not require manual adjustment of optimization parameters.

To represent the $3 \mathrm{D}$ orientation of the $\mathrm{AA}$ and FA of the MP joint, the 3D axes were projected to the $X O Z$ and $X O Y$ planes of the foot local anatomical coordinate system (see Figures 2C,D), where the angles $\alpha$ and $\beta$ were used to define the axis orientation with counter clockwise being positive based on the right-hand rule (see Figures 2C,D). In addition, to determine the 3D position of the AA and FA of the MP joint, the intersection point $\left(x_{a}, y_{a}\right)$ between the AA and the XOY plane of the foot local coordinate system, or 

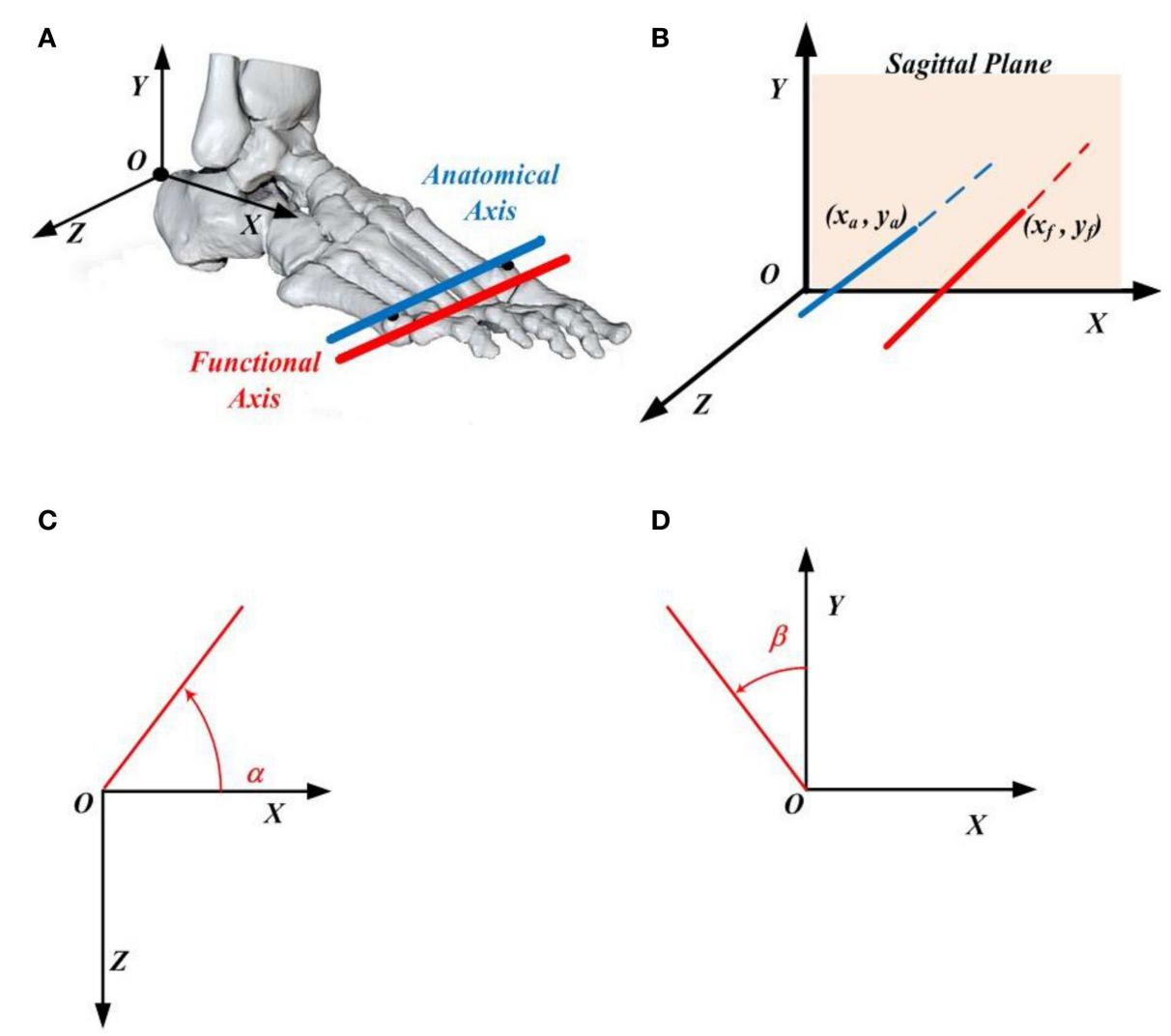

FIGURE 2 | (A) The functional axis (red) and anatomical axis (blue) of the metatarsophalangeal joint, where the anatomical axis is defined as the line connecting the first and fifth metatarsal heads. The origin of the foot local coordinate system situates on the upper ridge of the calcaneus bone. (B) The position of the functional axis is defined by its intersection point $\left(x_{f}, y_{f}\right)$ with the XOY plane of the foot coordinate system, whereas the location of the anatomical axis is determined by its intersection point $\left(x_{a}, y_{a}\right)$ with the XOY plane of the foot coordinate system. (C) Angle $\alpha$ made by the functional (or anatomical) axis with respect to the foot $X$ axis when projected to the $X O Z$ plane of the foot coordinate system. (D) Angle $\beta$ made by the functional (or anatomical) axis with respect to the foot $Y$ axis when projected to the XOY plane of the foot coordinate system. the intersection point $\left(x_{f}, y_{f}\right)$ between the FA and the XOY plane of the foot local coordinate system was used (see Figure 2B).

\section{DATA ANALYSIS}

The raw measured data were processed using GMAS software (Generalized Motion Analysis Software), a MATLAB based software package for 3D kinematic and kinetic analysis of biomechanical multi-body systems (Ren et al., 2005, 2008b). Trials with more than 10 consecutive missing frames were discarded. After the fillgap processing, the data were filtered using a low pass zero lag fourth-order Butterworth digital filter with a cut-off frequency of $6.0 \mathrm{~Hz}$.

Statistical analyses were conducted to investigate the $3 \mathrm{D}$ position and orientation differences between the AA and FA of the MP joint, and also the effect of walking speed on the AA and FA of the MP joint using SPSS 20.0 software (IBM, Armonk, New York, NY, USA). The effects of joint definition (AA or FA) and walking speed on joint position parameters $(x, y)$ and orientation parameters $(\alpha, \beta)$ were analyzed using analysis of variance (ANOVA) with repeated measurements using a linear mixed model approach taking into account intra- and inter-subject variability. The different joint definitions and walking speeds were the fixed effects, and subjects and trials were random effects. Differences between the two joint definitions (AA and FA) and between each pair of walking speeds were tested using Fisher's least significant difference (LSD) multiple comparison based on the least-squared means, probability by considering $p<0.05$ as statistically significant.

\section{RESULTS}

The measured data for all the six subjects were processed by using the method described in the preceding section. Figure 3 shows the mean and standard deviation values of the MP joint AA position $\left(x_{a}, y_{a}\right)$ and FA position $\left(x_{f}, y_{f}\right)$ defined in the XOY plane of the foot local coordinate system, where the origin of the coordinate system is at the upper ridge of the calcaneus bone (Ren et al., 2010). The position data of both AA and FA axes for all the six subjects (from subject A to subject F) at all three different walking speeds (slow, normal, and fast) are presented in Figure 3. It can be seen that the FA is consistently anterior to the AA for all subjects across all the three walking speeds with an average position difference of $27.3 \mathrm{~mm}$. In the inferior superior direction (along $Y$ axis), both FA and AA positions are close to the origin, and 


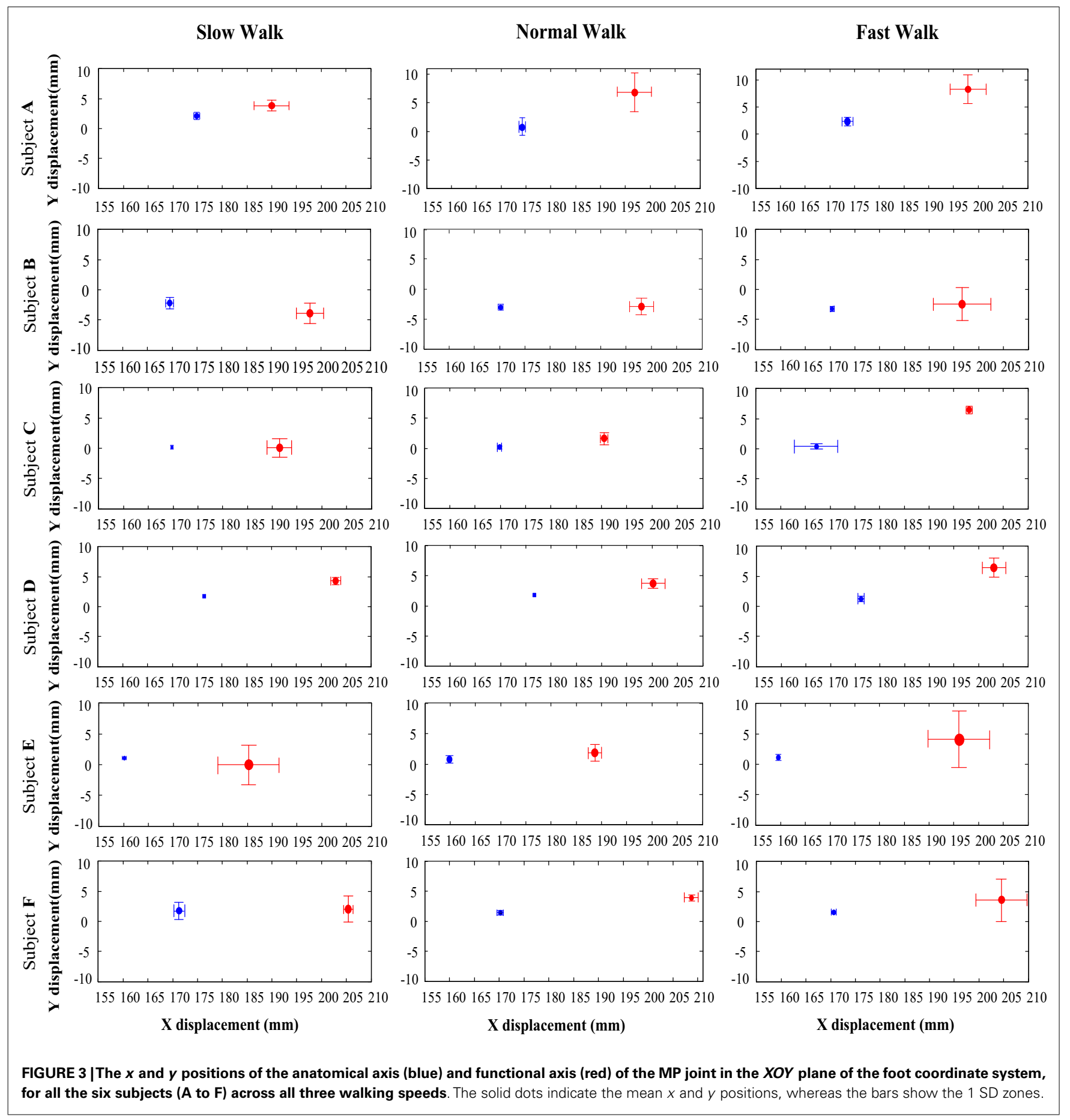

there is no consistent trend in the relative position between the two axes. From the results shown in Figure 3, it appears that the FA of MP joint moves toward a more anterior and superior position with increased walking speed though the displacement in the inferior superior direction (along $Y$ axis) is much less than the displacement in the posterior anterior direction (along $X$ axis). In contrast to the trend of the FA, it seems that there is no apparent change in the AA position with increased walking speed for all the subjects.
Figure 4 shows the mean and the 1 SD zone of the orientation angle $\alpha$ of both AA and FA made with respect to the $X$ axis in the $X O Z$ plane of the foot coordinate system for all the subjects at all three walking speeds. It can be seen that both AA and FA axes are close to the direction of the foot $Z$ axis. According to the right-hand rule, the positive angle $\alpha$ is measured starting from the $X$ axis in a counter clockwise direction. From Figure 4, it appears that the FA has an orientation more inclined to the foot $Z$ axis than the AA in the transverse plane for all the subjects. Also, it seems 


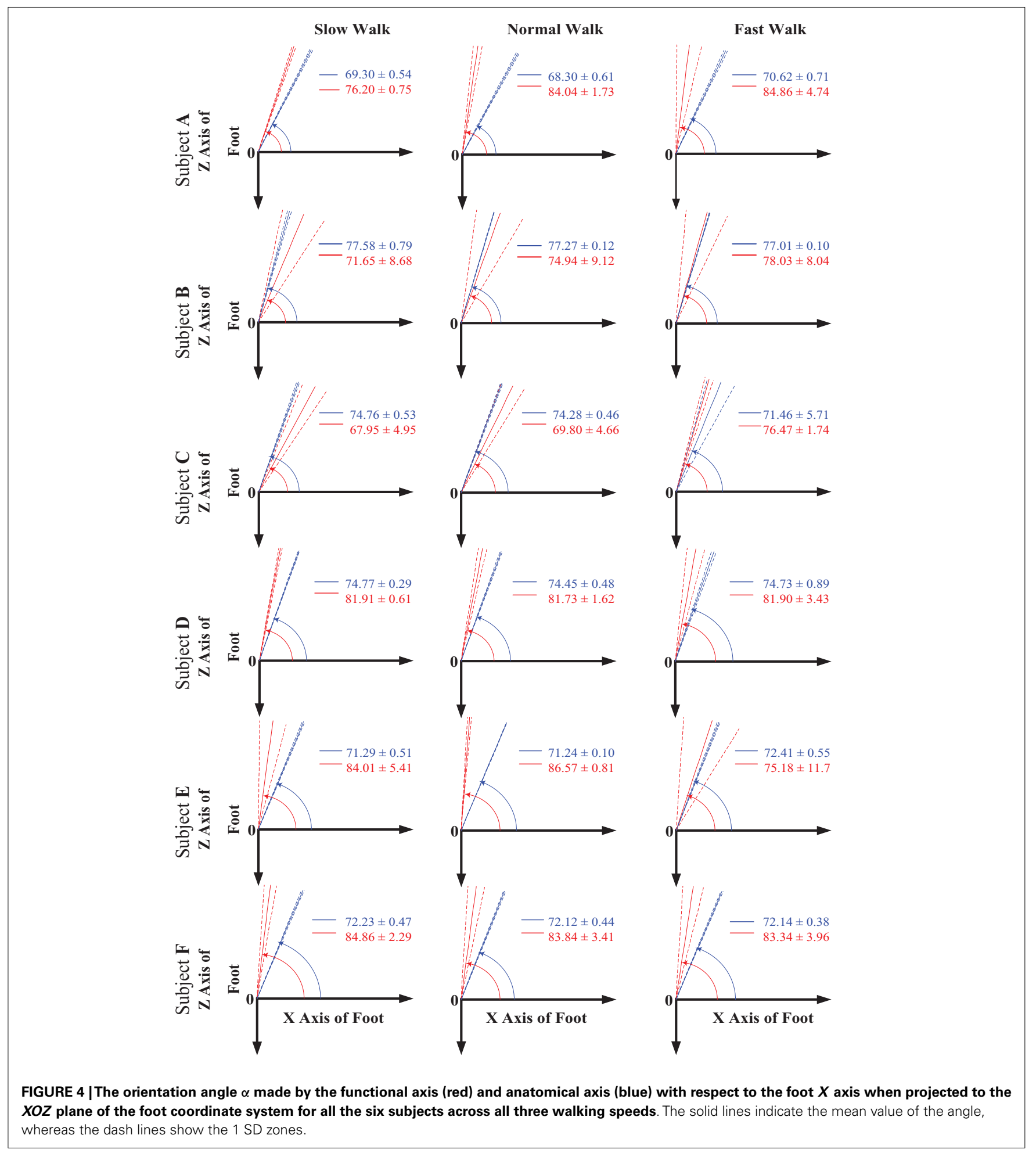

that the obliquity of both AA and FA does not change obviously with changing speed.

Similarly, Figure 5 shows the mean and the 1 SD zone of the orientation angle $\beta$ of both AA and FA made with respect to the $Y$ axis in the XOY plane of the foot coordinate system for all the subjects at all three walking speeds. According to the right-hand rule, the negative angle $\beta$ is measured starting from the $Y$ axis in a clockwise direction. It can be seen that the FA shows an apparently different orientation than the $\mathrm{AA}$ in the sagittal plane for all the subjects. The FA possesses a higher orientation angle $\beta$ than the AA leading to a FA direction more inclined to the foot $Y$ axis. From the results shown in Figure 5, it seems that the orientation angles 

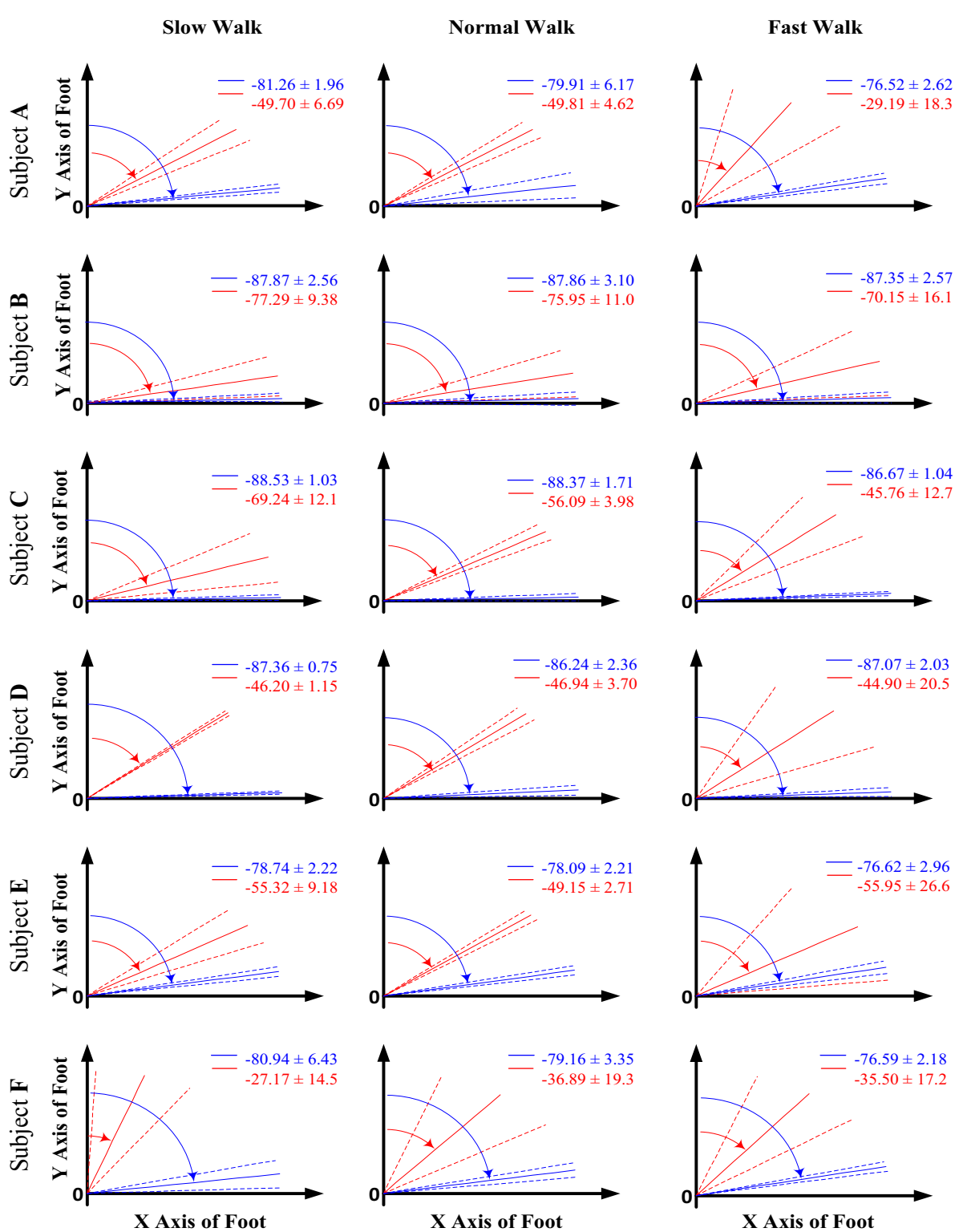

FIGURE 5 |The orientation angle $\beta$ made by the functional axis (red) and anatomical axis (blue) with respect to the foot $Y$ axis when projected to the XOY plane of the foot coordinate system for all the six subjects across all three walking speeds. The solid lines indicate the mean value of the angle, whereas the dash lines show the 1 SD zones.

in the sagittal plane for both AA and FA do not show particular changes with increased walking speed.

In Table 1, the statistical analysis result on the $3 \mathrm{D}$ position and orientation difference between the AA and FA of the MP joint is listed. The position parameters $\left(x_{a}, y_{a}\right)$ and $\left(x_{f}, y_{f}\right)$ are normalized by the foot length, defined as the distance between the upper ridge of the calcaneus bone and the midpoint between the first and fifth metatarsal heads. It can be seen that statistically significant differences were found for all the 3D orientation and position parameters across all three walking speeds, except for the vertical (in superior inferior direction) position parameter $y$ at slow walking speed. The functional rotation axis, FA of the MP joint has a more anterior and superior position than that of the AA, especially during normal and fast walking. This position difference is more significant in superior inferior direction than in anterior posterior direction. The FA of the MP joint shows an anteriorly more medial and more superior orientation than the AA, which is defined as the straight line connecting the first and fifth metatarsal heads. This joint orientation angle difference is more significant in the sagittal plane than in the transverse plane.

The statistical analysis result investigating the effect of walking speed on the 3D position and orientation of the FA is shown in Table 2. It can be seen that the joint position in the anterior posterior direction shows statistically significant difference when 
Table 1 | The result of the statistical analysis of the position and orientation difference between the functional axis (FA) and the anatomical axis (AA) of the MP joint.

\begin{tabular}{llcccc}
\hline & & $\begin{array}{c}\boldsymbol{x} \text { Normalized by } \\
\text { foot length }\end{array}$ & $\begin{array}{c}\boldsymbol{y} \text { Normalized by } \\
\text { foot length }\end{array}$ & $\boldsymbol{\alpha}$ (degree) & $\boldsymbol{\beta}$ (degree) \\
\hline Slow walk & FA & $1.1496 \pm 0.0424^{\mathrm{a}}$ & $0.0050 \pm 0.0193^{\mathrm{a}}$ & $77.8345 \pm 7.9035^{\mathrm{a}}$ & $-52.6132 \pm 24.7011^{\mathrm{a}}$ \\
& AA & $0.9989 \pm 0.0100^{\mathrm{b}}$ & $0.0042 \pm 0.0096^{\mathrm{a}}$ & $73.5031 \pm 2.7248^{\mathrm{b}}$ & $-84.2468 \pm 4.8971^{\mathrm{b}}$ \\
Normal walk & FA & $1.1619 \pm 0.0415^{\mathrm{a}}$ & $0.0153 \pm 0.0185^{\mathrm{a}}$ & $81.2593 \pm 6.4280^{\mathrm{a}}$ & $-51.0727 \pm 15.0268^{\mathrm{a}}$ \\
& AA & $0.9982 \pm 0.0104^{\mathrm{b}}$ & $0.0029 \pm 0.0098^{\mathrm{b}}$ & $72.8288 \pm 2.8084^{\mathrm{b}}$ & $-82.7051 \pm 5.2145^{\mathrm{b}}$ \\
Fast walk & FA & $1.1683 \pm 0.0390^{\mathrm{a}}$ & $0.0250 \pm 0.0250^{\mathrm{a}}$ & $80.0469 \pm 6.3455^{\mathrm{a}}$ & $-47.2030 \pm 23.4659^{\mathrm{a}}$ \\
& AA & $0.9942 \pm 0.0187^{\mathrm{b}}$ & $0.0028 \pm 0.0110^{\mathrm{b}}$ & $73.2755 \pm 3.0998^{\mathrm{b}}$ & $-82.4910 \pm 5.6568^{\mathrm{b}}$
\end{tabular}

Values are means \pm s.e.m. for all trials and all subjects. Identical letters indicate axis groups within a column do not differ significantly from each other ( $p>0.05)$.

Table 2 |The result of the statistical analysis of the effect of walking speed on the orientation and position of the functional axis (FA) of the MP joint.

\begin{tabular}{llcll}
\hline & $\begin{array}{c}\boldsymbol{x}_{\boldsymbol{f}} \text { Normalized by } \\
\text { foot length }\end{array}$ & $\begin{array}{c}\boldsymbol{y}_{\boldsymbol{f}} \text { Normalized by } \\
\text { foot length }\end{array}$ & $\boldsymbol{\alpha}$ (degree) & $\boldsymbol{\beta}$ (degree) \\
\hline Slow walk & $1.1496 \pm 0.0424^{\mathrm{a}}$ & $0.0050 \pm 0.0193^{\mathrm{a}}$ & $77.8345 \pm 7.9035^{\mathrm{a}}$ & $-52.6132 \pm 24.7011^{\mathrm{a}}$ \\
Normal walk & $1.1619 \pm 0.0415^{\mathrm{ab}}$ & $0.0153 \pm 0.0185^{\mathrm{b}}$ & $81.2593 \pm 6.4280^{\mathrm{a}}$ & $-51.0727 \pm 15.0268^{\mathrm{a}}$ \\
Fast walk & $1.1683 \pm 0.0390^{\mathrm{b}}$ & $0.0250 \pm 0.0250^{\mathrm{c}}$ & $80.0469 \pm 6.3455^{\mathrm{a}}$ & $-47.2030 \pm 23.4659^{\mathrm{a}}$
\end{tabular}

Values are means \pm s.e.m. for all trials and all subjects. Identical letters indicate speed groups within a column do not differ significantly from each other ( $p>0.05$ ).

Table 3 |The result of the statistical analysis of the effect of walking speed on the orientation and position of the anatomical axis (AA) of the MP joint.

\begin{tabular}{lcccc}
\hline & $\begin{array}{c}\boldsymbol{x}_{\boldsymbol{a}} \text { Normalized by } \\
\text { foot length }\end{array}$ & $\begin{array}{c}\boldsymbol{y}_{\mathbf{a}} \text { Normalized by } \\
\text { foot length }\end{array}$ & $\boldsymbol{\alpha}$ (degree) & $\boldsymbol{\beta}$ (degree) \\
\hline Slow walk & $0.9989 \pm 0.0100^{\mathrm{a}}$ & $0.0042 \pm 0.0096^{\mathrm{a}}$ & $73.5031 \pm 2.7248^{\mathrm{a}}$ & $-84.2468 \pm 4.8971^{\mathrm{a}}$ \\
Normal walk & $0.9982 \pm 0.0104^{\mathrm{a}}$ & $0.0029 \pm 0.0098^{\mathrm{b}}$ & $72.8288 \pm 2.8084^{\mathrm{a}}$ & $-82.7051 \pm 5.2145^{\mathrm{ab}}$ \\
Fast walk & $0.9942 \pm 0.0187^{\mathrm{a}}$ & $0.0028 \pm 0.0110^{\mathrm{ab}}$ & $73.2755 \pm 3.0998^{\mathrm{a}}$ & $-82.4910 \pm 5.6568^{\mathrm{b}}$
\end{tabular}

Values are means \pm s.e.m. for all trials and all subjects. Identical letters indicate speed groups within a column do not differ significantly from each other ( $p>0.05)$.

walking speed changes from slow to fast. The FA moves forwards toward a more anterior position with increased speed. More significant change can be seen in the superior inferior direction, the FA moves upwards toward a more superior position appreciably when walking speed increases. No statistically significant effects are found on the FA orientation angles in both the transverse $(\alpha)$ and sagittal $(\beta)$ planes when walking speed increases from self-selected low to self-selected fast.

Similarly, Table 3 shows the statistical analysis result examining the effect of walking speed on the $3 \mathrm{D}$ position and orientation of the AA of the MP joint. No statistically significant change is found in the AA position along the anterior posterior direction. When walking speed increases to normal, the AA position in the superior inferior direction moves apparently toward the inferior direction. There is no statistically significant effect found on the AA orientation angle in the transverse plane $(\alpha)$ with changing speed, whereas a slight increase of the AA orientation angle in the sagittal plane $(\beta)$ was found when walking speed increases from slow to fast.

\section{DISCUSSION}

The objective of this study is to investigate the 3D orientation and location of the functional joint axis of the MP joint during walking at different speeds. Here, the functional joint axis of the MP joint is defined as the relative rotational axis between the phalanx segments and the hindfoot in the stance phase of walking. In the previous studies, the MP joint was normally defined as a line connecting the first (or second) and fifth metatarsal heads (Boonpratatong and Ren, 2010; Smith et al., 2012), and little is known about the realistic position of the functional joint axis of the MP joint.

Our results show that the $3 \mathrm{D}$ orientation and position of the FA of the MP joint is close to the AA defined by the line connecting first and fifth metatarsal heads. However, for the subjects tested in this study, there are some statistically significant differences between the FA and AA. The FA remains anterior to the AA with an average distance about $16 \%$ of the foot length across all walking speeds. In the vertical direction, the FA is superior to the AA with an average distance about $2 \%$ of the foot length during normal and fast walking, whereas, the FA shows a higher obliquity 
than AA with an anteriorly more medial and superior orientation. This suggests that using the AA to represent the MP joint may result in overestimated MP joint moment and power and also underestimated muscle moment arms for MP extensor muscles.

It was found that walking speed has statistically significant effect on the position of the FA though the FA orientation remains unchanged with varying speed. When walking speed increases, the FA moves forwards and upwards toward a more anterior and more superior position. This joint axis shift toward the ground reaction force vector in the late stance of walking will result in decreased moment arm of ground reaction force and also simultaneously increased moment arm of MP extensor muscles, and hence will increase the EMA of MP extensor muscles (Biewener, 1989, 1990, 2003; Biewener et al., 2004; Winter, 2005). In addition, a forward shift of the MP joint axis will result in an increased lever distance to the ankle joint, and may moderate the angular velocity increase with increasing walking speed. This may help to maintain the contraction velocity of the MP extensor muscles in the power optimal region (Biewener, 2003). It appears that a variable gear mechanism also exists in the MP joint especially in the late stance of walking (Carrier et al., 1994).

This study has some limitations. A single hinge type joint was assumed to represent the relative motion between the phalanges and the rearfoot throughout the stance phase of walking. As suggested by previous studies, two different axes may exist at the MP level during locomotion ( Bojsen-Møller, 1978; Bojsen-Møller and Lamoreux, 1979). Our future works will involve the investigation of the 3D positions and transition of multiple MP axes. In addition, two rigid bodies (phalanges and hindfoot) were assumed to represent the motion of the foot complex by using multiple marker clusters in the gait measurement. However, in reality, some appreciable relative motions may occur between phalanges or the hindfoot bones. Thus, more complicated models may be needed in the future to better understand the 3D kinematics at MP joint. Furthermore, bone-pin markers rather than skin mounted markers may help to reduce the skin artifact involved in the gait measurement (Nester et al., 2007).

\section{CONCLUSION}

This study reveals many factors at the distal end of the foot, which may contribute to the locomotor function of the human foot complex. This includes not only the obliquely oriented functional rotation axis but also the relative position of the axis with respect to the hindfoot bones. The position of the FA of the MP joint is found to be anterior and superior to the AA with higher obliquity. Furthermore, with increasing speed the FA shifts more anterior. This forward and upward shift of the FA with increasing walking speed may help to moderate the muscular effort, maximize the locomotor efficiency, and reduce the risk of injury. This study may help us to better understand the contribution of the intrinsic foot structure to the propulsive function of the foot during locomotion at different speeds. Furthermore, this may also help in improving the design of sports and therapeutic footwears, prosthetic lower limbs, and robotic legs.

\section{ACKNOWLEDGMENTS}

The assistance and support of John R. Hutchinson on measurement data collection have been valuable.

\section{REFERENCES}

Apkarian, J., Naumann, S., and Cairns, B. (1989). A three dimensional kinematic and dynamic model of the lower limb. J. Biomech. 22, 143-155. doi:10.1016/ 0021-9290(89)90037-7

Biewener, A. A. (1989). Scaling body support in mammals: limb posture and muscle mechanics. Science 245, 45-48. doi:10.1126/science.2740914

Biewener, A. A. (1990). Biomechanics of mammalian terrestrial locomotion. Science 250, 1097-1103. doi:10.1126/science.2251499

Biewener, A. A. (2003). Animal Locomotion. New York: Oxford University Press Inc.

Biewener, A. A., Farley, C. T., Roberts, T. J., and Temaner, M. (2004). Muscle mechanical advantage of human walking and running: implications for energy cost. J. Appl. Physiol. 97, 2266-2274. doi:10.1152/japplphysiol.00003.2004

Bojsen-Møller, F. (1978). "The human foot a two speed construction," in International Series of Biomechanics, Vol. 6, eds E. Asmussen and K. Jorgensen. 261-266.

Bojsen-Møller, F., and Lamoreux, L. (1979). Significance of free dorsiflexion of the toes in walking. Acta Orthop. Scand. 50, 471-479. doi:10.3109/ 17453677908989792

Boonpratatong, A., and Ren, L. (2010). The human ankle-foot complex as a multiconfigurable mechanism during the stance phase of walking. J. Bionic Eng. 7, 211-218. doi:10.1016/S1672-6529(10)60243-0

Cappozzo, A., Catani, F., Della, C. U., and Leardini, A. (1995). Position and orientation in space of bones during movement: anatomical frame definition and determination. Clin. Biomech. 10, 171-178. doi:10.1016/0268-0033(95)91394-T

Carrier, D. R., Heglund, N. C., and Earls, K. D. (1994). Variable gearing during locomotion in the human musculoskeletal system. Science 265, 651-653. doi:10.1126/science.8036513

Carson, M. C., Harrington, M. E., Thompson, N., O'Connor, J. J., and Theologis, T. N. (2001). Kinematic analysis of multi-segment foot model for research and clinical applications: a repeatability analysis. J. Biomech. 34, 1299-1307. doi:10.1016/S0021-9290(01)00101-4

Gamage, S. S., and Lasenby, J. (2002). New least squares solutions for estimating the average centre of rotation and the axis of rotation. J. Biomech. 35, 87-93. doi:10.1016/S0021-9290(01)00160-9

Gefen, A., Megido-Ravid, M., Itzchak, Y., and Arcan, M. (2000). Biomechanical analysis of the three-dimensional foot structure during gait: a basic tool for clinical applications. J. Biomech. 122, 630-639. doi:10.1115/1.1318904

Graf, E. S., Wright, I. C., and Stefanyshyn, D. J. (2012). Effect of relative marker movement on the calculation of the foot torsion axis using a combined Cardan angle and helical axis approach. Comput. Math. Methods Med. 2012, 368050. doi:10.1155/2012/368050

Jenkyn, T. R., and Nicol, A. C. (2007). A multi-segment kinematic model of the foot with a novel definition of forefoot motion for use in clinical gait analysis during walking. J. Biomech. 40, 3271-3278. doi:10.1016/j.jbiomech.2007.04.008

Ker, R. F., Bennett, M. B., Bibby, S. R., Kester, R. C., and Alexander, R. M. (1987). The spring in the arch of the human foot. Nature 325, 147-149. doi:10.1038/ $325147 \mathrm{a} 0$

Leardini, A., Benedetti, M. G., Catani, F., Simoncini, L., and Giannini, S. (1999). An anatomically based protocol for the description of foot segment kinematics during gait. Clin. Biomech. 14, 528-536. doi:10.1016/S0268-0033(99)00008-X

MacWilliams, B. A., Cowley, M., and Nicholson, D. E. (2003). Foot kinematics and kinetics during adolescent gait. Gait Posture 17, 214-224. doi:10.1016/S09666362(02)00103-0

Mann, R. A., and Hagy, J. L. (1979). The function of the toes in walking, jogging and running. Clin. Orthop. Relat. Res. 142, 501-510.

Nester, C. J., Liu, A., Ward, E., Howard, D., Cocheba, J., Derrick, T., et al. (2007). In vitro study of foot kinematics using a dynamic walking cadaver model. J. Biomech. 40, 1927-1937. doi:10.1016/j.jbiomech.2006.09.008

Pohl, M. B., Messenger, N., and Buckley, J. G. (2007). Forefoot, rearfoot and shank coupling: effect of variations in speed and mode of gait. Gait Posture 25, 295-302. doi:10.1016/j.gaitpost.2006.04.012

Qian, Z., Ren, L., Ding, Y., Hutchinson, J. R., and Ren, L. (2013). A dynamic finite element analysis of human foot complex in the sagittal plane during level walking. PLoS ONE 8:e79424. doi:10.1371/journal.pone.0079424

Ren, L., Howard, D., Ren, L., Nester, C., and Tian, L. (2008a). A phase-dependent hypothesis for locomotor functions of human foot complex. J. Bionic Eng. 5, 175-180. doi:10.1016/S1672-6529(08)60022-0

Ren, L., Jones, R. K., and Howard, D. (2008b). Whole body inverse dynamics over a complete gait cycle based only on measured kinematics. J. Biomech. 41, 2750-2759. doi:10.1016/j.jbiomech.2008.06.001 
Ren, L., Howard, D., Ren, L., Nester, C., and Tian, L. (2010). A generic analytical foot rollover model for predicting translational ankle kinematic in gait simulation studies. J. Biomech. 43, 194-202. doi:10.1016/j.jbiomech.2009.09.027

Ren, L., Jones, R. K., and Howard, D. (2005). Dynamic analysis of load carriage biomechanics during level walking. J. Biomech. 38, 853-863. doi:10.1016/j.jbiomech. 2004.04.030

Ren, L., Qian, Z., and Ren, L. (2014). Biomechanics of musculoskeletal system and its biomimetic implications: a review. J. Bionic Eng. 11, 159-175. doi:10.1016/S1672-6529(14)60033-0

Scott, S. H., and Winter, D. A. (1993). Biomechanical model of the human foot: kinematics and kinetics during the stance phase of walking. J. Biomech. 26, 1091-1104. doi:10.1016/S0021-9290(05)80008-9

Smith, G., Lake, M., Lees, A., and Worsfold, P. (2012). Measurement procedures affect the interpretation of the metatarsophalangeal joint function during accelerated sprinting. J. Sports Sci. 30, 1521-1527. doi:10.1080/02640414.2012.713501

Stefanyshyn, D. J., and Nigg, B. M. (1997). Mechanical energy contribution of the metatarsophalangeal joint to running and sprinting. J. Biomech. 20, 1081-1085. doi:10.1016/S0021-9290(97)00081-X

Stefanyshyn, D. J., and Nigg, B. M. (1998). Contribution of the lower extremity joints to mechanical energy in the running vertical jumps and running long jumps. J. Sports Sci. 16, 177-186. doi:10.1080/026404198366885
Winter, D. A. (2005). The Biomechanics and Motor Control of Human Movement, 3rd Edn. New Jersey: John Wiley and Sons, Inc.

Conflict of Interest Statement: The authors declare that the research was conducted in the absence of any commercial or financial relationships that could be construed as a potential conflict of interest.

Received: 30 August 2014; accepted: 01 December 2014; published online: 15 December 2014.

Citation: Raychoudhury S, Hu D and Ren L (2014) Three-dimensional kinematics of the human metatarsophalangeal joint during level walking. Front. Bioeng. Biotechnol. 2:73. doi: 10.3389/fbioe.2014.00073

This article was submitted to Biomechanics, a section of the journal Frontiers in Bioengineering and Biotechnology.

Copyright (C) 2014 Raychoudhury, Hu and Ren. This is an open-access article distributed under the terms of the Creative Commons Attribution License (CC BY). The use, distribution or reproduction in other forums is permitted, provided the original author(s) or licensor are credited and that the original publication in this journal is cited, in accordance with accepted academic practice. No use, distribution or reproduction is permitted which does not comply with these terms. 